\title{
A comparison of maternal effects and current environment on vital rates of Aphis nerii, the milkweed-oleander aphid
}

\author{
CARALYN B. ZEHNDER ${ }^{1}$ and MARK D. HUNTER ${ }^{2}{ }^{1}$ Institute of Ecology, University of \\ Georgia, Athens, U.S.A. and ${ }^{2}$ Department of Ecology and Evolutionary Biology \& School of Natural Resources and Environment, \\ University of Michigan, Ann Arbor, U.S.A.
}

\begin{abstract}
Non-Mendelian maternal effects, the effects of maternal phenotype or environment on offspring phenotype, have been documented in numerous taxa. By affecting offspring vital rates (birth, death, and movement), maternal effects have the potential to influence population dynamics. However, relatively few studies have directly linked maternal phenotype or environment to offspring vital rates. Additionally, even fewer studies have compared the magnitude of across-generation effects (i.e. maternal effects) to within-generation effects.

2. Because of their telescoping generations, aphids can be strongly influenced by maternal effects. The effects of maternal density and maternal host-plant species on offspring survival, fecundity, and alate formation were investigated experimentally in Aphis nerii, the milkweed-oleander aphid.

3. Additionally, the relative strength of maternal effects were compared with those operating within a generation. Therefore, in another set of experiments, the effects of current density and host-plant species (within-generation effects) on aphid vital rates were examined.

4. While maternal effects were present, within-generation effects were much stronger and more strongly influenced aphid vital rates. Within a generation, aphids exhibited density-dependent survival, fecundity, and alate formation and these effects varied among host-plant species.

5. These results indicate that while maternal effects have the potential to affect population dynamics, this potential is not always met. Additionally, the current environment, not the environment of previous generations, more strongly impacts population dynamics.
\end{abstract}

Key words. Aphis nerii, density dependence, maternal effects, plant-insect interactions, plant quality.

\section{Introduction}

Non-Mendelian maternal effects describe the effect of a mother's phenotype or environment on offspring phenotype (Mousseau \& Fox, 1998; Agrawal et al., 1999; Beckerman et al., 2002). Cues in the maternal environment can affect offspring size (Wainhouse et al., 2001; Fischer et al., 2003; Plaistow et al., 2004), survival (Hockham et al., 2001; Wainhouse et al., 2001), development

Correspondence: Caralyn B. Zehnder, Institute of Ecology, University of Georgia, Athens, GA 30602-2202, U.S.A. E-mail: zehnder@uga.edu rate (Rossiter, 1991a; Fox et al., 1995; Benton et al., 2005), fecundity (Rossiter, 1991b; Hockham et al., 2001; Benton et al., 2005), movement (Islam et al., 1994; Diss et al., 1996), and defences (Agrawal, 1999). While it is clear that maternal effects are pervasive, their potential ecological impacts and relative importance are not well understood and warrant further investigation (Beckerman et al., 2002).

Maternal effects have the potential to influence population dynamics (Rossiter, 1991a; Ginzburg \& Taneyhill, 1994; Benton et al., 2001; Beckerman et al., 2002; Hunter, 2002; Benton et al., 2005; Kendall et al., 2005). By connecting the maternal environment or phenotype to offspring phenotype, maternal

(C) 2007 The Authors Journal compilation (C) 2007 The Royal Entomological Society 
effects introduce a time lag that can cause a delay in densitydependent processes (Rossiter, 1994) leading to population cycles (Berryman, 1999). Models with maternal effects as the mechanism underlying the time lag in density-dependence have shown that maternal effects generally cause increased population fluctuation (Benton et al., 2001).

Plant quality is a broad term that encompasses any physical, chemical, or biological traits of plants (e.g. size and structure, nutritional value, secondary compounds, and phenology) that influence herbivore preference or performance. Variation in host-plant quality influences insect herbivore survival (Haggstrom \& Larsson, 1995; Lill \& Marquis, 2001; Ladner \& Altizer, 2005), development time (Haggstrom \& Larsson, 1995; Tsai \& Wang, 2001; Wheeler, 2001; Ladner \& Altizer, 2005), fecundity (Rossiter, 1988; Tsai \& Wang, 2001), and the strength of density-dependence (Agrawal, 2004).

For parthenogenetic herbivores, different clones may vary in their responses to different host plants. For example, studies of pea aphids on alfalfa and red clover have shown that clones collected from these two hosts prefer to feed on their own 'home' host (Via, 1991) and that pea aphids recognise host specific chemical stimulants (Del Campo et al., 2003). In Aphis nerii, clonal variation in the proportion of alates produced has been correlated with the degree of habitat permanence (Groeters \& Dingle, 1989).

Aphids are ideal organisms with which to study maternal effects. Aphids have telescoping generations, which means that within a single aphid there are embryos within embryos. This large generational overlap, granddaughters are present within their grandmothers, could lead to strong maternal effects (Dixon, 1998). Additionally, aphids reproduce parthenogenetically for much of their life cycle and some species never have sexual reproduction (Dixon, 1998). Mothers are genetically identical to their daughters and sisters, and there is no genetic recombination (Hales et al., 2002). The lack of genetic variation across generations is ideal for focusing on non-genetic maternal effects. Furthermore, the lack of genetic variation prevents selection from taking place during experiments. An additional benefit of working on aphids is their short generation time, which allows for a high potential correlation between maternal environment and offspring environment. Maternal effects are known to be important in aphids, especially in the production of different morphs (Dixon, 1998). Typically, cues in the maternal environment such as crowding (Hall \& Ehler, 1980), low temperature (Nunes \& Hardie, 1999), decline in plant quality (Wiktelius, 1992), changing photoperiod (Hardie \& Lees, 1983), or the presence of natural enemies (Dixon \& Agarwala, 1999) lead to the production of alates (winged aphids). Similar cues lead to the production of sexual or diapausing morphs in certain species (Lees, 1963).

Variation in insect density, plant quality and insect clone can all potentially affect vital rates both across and within-generations. Therefore the question is: which of these ecological factors is most important and when do they operate? More specifically, it was hypothesised that maternal host-plant species and maternal density would strongly influence offspring vital rates. Additionally, it was hypothesised that host-plant species and aphid clone would affect within-generation density-dependent survival, fecundity, and alate productive of $A$. nerii.

\section{Methods}

\section{Study system}

Aphis nerii Boyer de Fonscolombe (Hemiptera: Aphididae), the milkweed-oleander aphid, is an aposematic phloem-feeding specialist of milkweed (Asclepias spp.) and oleander, Nerium oleander L. (Apocynaceae) that reproduces parthenogenetically.

In August 2003, three A. nerii individuals were collected from geographically distinct locations (Atlanta, Georgia; Augusta, Georgia; and Gainesville, Florida). Each of these is referred to as a separate clone (i.e. clone Emory, Augusta, and Florida). An aphid colony was initiated from one individual from each location, leading to three laboratory colonies. Separate colonies of these aphid clones were kept at low densities on Asclepias syriaca in the laboratory. The colonies were kept in the same growth chamber in which all the experiments were run (described below) and experienced the same light and temperature regime. An explicit comparison of these clones was made only in experiment 3 (below). The genus Asclepias is composed of predominately herbaceous perennials that contain a class of compounds known as cardenolides or cardiac glycosides. There is a large degree of variation in cardenolide concentration among Asclepias species both qualitatively and quantitatively (Malcolm et al., 1989; Agrawal, 2004).

All research was conducted in a temperature and light controlled walk-in growth chamber. Grow lights on timers (LD 16:8 h) provided heat and light; daytime and night-time temperatures were $34 \pm 2.4{ }^{\circ} \mathrm{C}$ and $24 \pm 0.48{ }^{\circ} \mathrm{C}$ respectively. Asclepias seeds were obtained from a seed distributor (Butterfly Encounters), grown in Farfard 3B soil with Osmocote time-release fertiliser (14-14-14, N:P:K) and watered as needed. In all experiments, plants were assigned randomly to treatments and rotated daily to homogenise any environmental gradients within the growth chamber. Figure 1 summarises the methods used in each experiment, described below.

Experiment 1: maternal host-plant effects. This experiment was designed to determine if the host-plant species of the maternal generation affects offspring vital rates; all variables except maternal host-plant species were held constant. Because aphids have telescoping generations, it was thought that the maternal host-plant effect would be stronger if multiple previous generations were on different host-plant species. Therefore, aphids were reared on different host-plant species for seven generations and then the eighth generation was on a common host-plant species. Seven Asclepias species were chosen: A. exaltata, A. incarnata, A. speciosa, A. sullivantii, A. syriaca, A. tuberosa, and A. viridis. These species encompass a range of variation in plant quality traits (Agrawal, 2004). At the start of the experiment, January 2005, all seedlings were 4 weeks old and approximately the same size. There were seven replicates of each host-plant species. A first-instar aphid from the clone Florida colony was placed on each plant and the first generation developed. There was no movement of aphids among plants. When the first generation started to reproduce, all but one offspring was removed from the plant. Mass and age (in days) at maturity was recorded for each generation. This continued for seven generations. Finally, when the seventh generation started to reproduce, their 

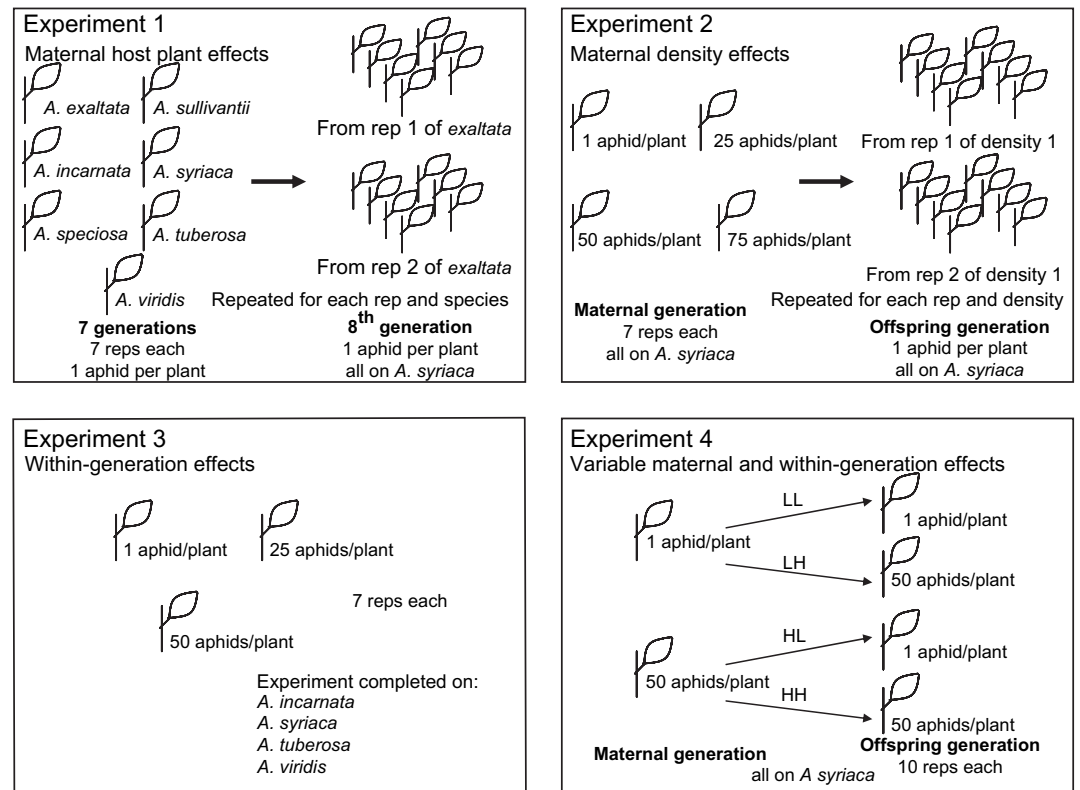

Fig. 1. Summary of experiments used to compare the relative importance of maternal and within-generation effects on the vital rates of Aphis nerii. rep, replicate. first seven offspring were removed from the plant and each offspring was placed individually on an A. syriaca seedling. Daily observations were made of survival and fecundity in the offspring generation. Age, mass, and aphid morph (alate or apterae) was recorded when the aphids reached maturity. Additionally, all offspring produced were removed (the offspring's offspring) after daily counts. These observations continued until all aphids died (approximately 30 days).

Experiment 2: maternal density effects. This experiment was designed to determine if maternal density influences offspring vital rates; all variables were held constant except for maternal density. In February 2004, the maternal generation was established at four densities: 1, 25, 50, and 75 aphids per plant, with each density replicated seven times. These densities represent natural aphid abundances (Hall \& Ehler, 1980). First-instar clone Florida aphids were placed on 4-week-old $A$. syriaca seedlings at the appropriate density, and each plant was caged to prevent aphids in the high density treatments from walking off the plants. Cages were constructed from 710-ml Ziploc containers and organza netting over wire frames. When the maternal generation started to reproduce, the first eight offspring were removed and placed individually on an A. syriaca seedling. There were a total of 224 aphids in the offspring generation (four maternal densities $\times$ seven replicates (mothers) per density $\times$ eight offspring). Daily observations were made of survival and fecundity in the offspring generation. Aphid morph was recorded when the aphids reached maturity. As before, all offspring produced were removed (the offspring's offspring) to keep densities constant. These observations continued until all aphids died (approximately 30 days).

Experiment 3: within-generation effects. Laboratory experiments were performed to examine density-dependent vital rates of A. nerii on four Asclepias species. These experiments investigated within-generation effects, not maternal effects. It was hypothesised that $A$. nerii would exhibit density-dependent survival, fecundity, and alate formation, and that these responses would vary among aphid clones and host-plant species. Four Asclepias species (A. tuberosa, A. viridis, A. incarnata, and A. syriaca) were chosen to represent a range of plant quality traits. The four species vary in foliar carbon:nitrogen ratios, trichome density (Agrawal, 2004), and cardenolide content (Malcolm et al., 1989; Agrawal, 2004).

The identical experimental design was repeated on the four host-plant species (A. tuberosa: June 2004, A. viridis: July 2004, A. incarnata: October 2004, and A. syriaca: November 2004). For each experiment, three aphid densities (1, 25, and 50 aphids per plant) were established on 1-month-old Asclepias seedlings. Initially, five adult aphids were placed on all experimental plants and left to reproduce on the plants overnight. They were then removed and their offspring thinned out to the appropriate density. This was repeated for all three aphid clones, except for the experiment on A. syriaca for which only the Emory and Florida clones were used because there were not enough aphids from the Augusta clone at the start of the experiment. Each density was replicated seven times, for a total of 63 plants per experiment (three densities $\times$ three clones $\times$ seven replicates $=63$ plants). Each plant was caged. Daily counts were made of the number of aphids surviving and number of offspring produced. All offspring were removed daily. This continued until all of the aphids died (approximately 30 days). When the aphids reached maturity, mass and proportion alate were recorded. Using a paintbrush, one aphid (on the low density treatment) or five aphids were removed and individually weighed on a Mettler Toledo balance (Mettler Toledo, Im Langacher, Switzerland, max. $2.1 \mathrm{~g}, d=0.1 \mu \mathrm{g}$ ). Then the aphids were placed carefully back on the plant. Because of time and space limitations, we could not examine all host plants at the same time. However, photoperiod, temperature, and other environmental conditions remained constant in the growth chamber over the course of all experiments. 
Experiment 4: variable maternal and offspring density effects. Results from the previous experiments led us to test the hypothesis that the expression of maternal effects depends on the offspring's environment. In April 2005, using aphids from a single clone (Florida), two densities were established in the maternal generation: one aphid per plant and 50 aphids per plant. There were 10 replicates of the high density treatment and 40 replicates of the low density treatment. Many more low density plants were needed to ensure that there were enough aphids to establish the high density treatment in the next generation. Their offspring were either put on plants at low density (L: one aphid per plant) or high density (H: 50 aphids per plant). This led to four possible combinations: $\mathrm{H}_{\text {maternal }} \mathrm{H}_{\text {offspring }}, \mathrm{H}_{\text {maternal }} \mathrm{L}_{\text {offspring }}$, $\mathrm{L}_{\text {maternal }} \mathrm{H}_{\text {offspring }}, \mathrm{L}_{\text {maternal }} \mathrm{L}_{\text {offspring }}$. For the low density offspring, one aphid was randomly chosen from the appropriate maternal density. For the high density offspring, 50 aphids were chosen from among multiple maternal plants. Each combination was replicated 10 times for a total of 40 experimental plants in the offspring generation. Each plant was caged. Daily counts were made of the number of aphids surviving and number of offspring produced. All offspring (the offspring's offspring) were removed daily. This continued until all of the aphids died. When the aphids reached maturity, mass and proportion alate was recorded. As before, when they reach maturity, aphids were removed, weighed, and returned to their plant to continue reproducing.

\section{Analyses}

Data were analysed using SAS 8.2 for Windows and the residuals of the ANOVA models were tested for normality. Data sets whose residuals failed to meet the assumptions of normality were transformed and reanalysed. Proportion surviving and proportion alate data were arcsine square root transformed, and, when necessary, other data were log transformed.

Maternal effects data (experiments 1 and 2) were analysed using a nested ANOvA (PROC GLM) with either host-plant species or density as the treatment effect and maternal ID nested with species or density. Response variables were number of days surviving, total fecundity, age at maturity, and mass at maturity (for experiment 1 only). If there was a significant effect of maternal environment on offspring survival, fecundity or age at maturity, then aphid instantaneous rate of increase $r$ was calculated using Euler's equation. Specifically, daily survivorship and fecundity were used to calculate generation time and estimate $r$. Then Euler's equation was iterated to produce a more exact estimate (Gotelli, 2001).

Within-generation effects on survival and fecundity (experiment 3) were analysed using the repeated measures framework of PROC MIXED with a Type 1 autoregressive model (Littell et al., 1998). To investigate within-generation host-plant effects (also experiment 3), all the within-generation effects experiments were combined in a single model. In this case, total fecundity, lifespan, mass, age at maturity, and proportion alate data were analysed using a three-way ANOVA with host plant, density, and clone as the treatment effects. A repeated measures framework was not used for this analysis because the response variables were all determined at the end of the experiment and not measured over time. Because the experiments with different host plants were conducted in different months (above), hostplant effects are confounded with any uncontrolled environmental variation among experiments. Host-plant comparisons in experiment 3 should therefore be viewed with caution.

Data from the final experiment were analysed using a twoway ANOVA with maternal density and offspring density as the two main effects and total fecundity, lifespan, mass, age at maturity, and proportion alate as response variables.

\section{Results}

\section{Experiment 1}

There was no effect of maternal host-plant species on offspring survival (nested ANOvA, $F_{6.38}=0.52, P=0.792$ ), fecundity (nested ANOVA, $F_{6,38}=0.56, P=0.758$ ), mass at maturity (nested ANOVA, $F_{6,38}=1.66 P=0.157$ ), or age at maturity (nested ANOVA, $F_{6,38}=1.43, P=0.229$ ) (data not shown). No alates were produced.

\section{Experiment 2}

Maternal density affected offspring development rate. Mothers in the lowest density treatment (one aphid per plant) produced offspring that matured on average nine hours (6.31 \pm 0.08 days) faster than those from mothers at higher densities (pooled mean for densities 25, 50, and 75: 6.9 \pm 0.05 days; nested ANOVA, $F_{3,24}=4.88, P=0.009$ ). Aphid instantaneous rate of increase $r$ decreased as maternal density increased [maternal density $($ aphids/plant $)=r$ (aphids/aphids $\times$ day): $1=0.67$, $25=0.62,50=0.613,75=0.605]$. There was no effect of maternal density on offspring survival (nested ANOVA, $F_{3,24}=$ $2.44, P=0.089$ ) or total fecundity (nested AnOva, $F_{3,24}=1.13$, $P=0.357$ ) (data not shown). As in the first experiment, no alates were produced.

\section{Experiment 3}

Within-generation effects on A. nerii vital rates were strong. Aphids exhibited density-dependent survival, fecundity, and alate formation. Rates of survival varied with density on all four host-plant species. (Fig. 2a-d Proc Mixed; A. tuberosa: density $\times$ date $F_{54,1572}=4.77, P<0.0001 ;$ A. incarnata: density $\times$ date $F_{52,1440}=2.42, P<0.0001 ;$ A. viridis: density $\times$ date $F_{64,1731}=$ $6.15, P<0.0001 ;$ A. syriaca: density $\times$ date $F_{54,990}=2.64 P<$ $0.0001)$. Additionally, density influenced patterns of fecundity over time on all host-plant species (Fig. 3a-d: A. tuberosa: density $\times$ date $F_{48,432}=21.10, P<0.0001 ;$ A. incarnata: density $\times$ date $F_{54,1494}=2.66, P<0.0001 ;$ A. viridis: density $\times$ date $F_{52,1412}=20.11, P<0.0001 ;$ A. syriaca: density $\times$ date $\left.F_{42,783}=3.5, P<0.0001\right)$. On A. tuberosa, clone Augusta reached its peak reproductive output earlier than the other aphid clones (data not shown: clone $\times$ date $F_{48,840}=1.89, P<$ $0.0003)$. The clone $\times$ date interaction was not significant on any of the other host-plant species. 

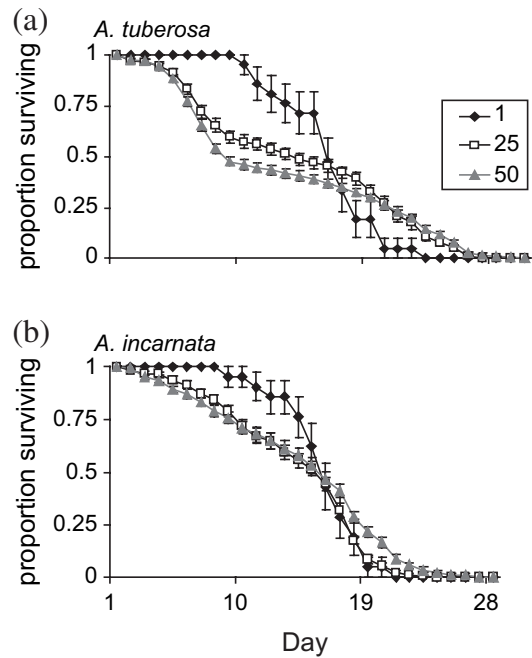

(c)

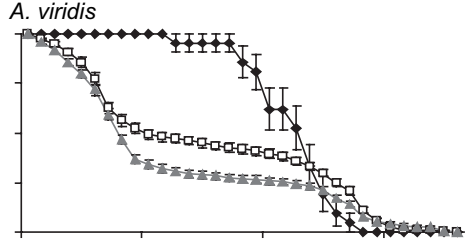

(d)

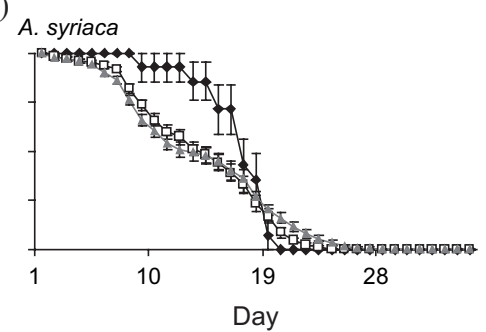

Fig. 2. Effects of density $(1,25,50$ aphids per plant) on Aphis nerii survival on four Asclepias species (a-d). Because aphid clone did not influence survival, results from the three clones were combined. Each point is the mean of 21 replicates \pm standard error.
In a larger model that included all four host-plant species, the expression of density-dependence on aphid vital rates varied among Asclepias species. Density-dependent reductions in fecundity were strongest on A. viridis (Fig. $4 \mathrm{a}$, host $\times$ density: $\left.F_{6,196}=15.24, P<0.0001\right)$. Similarly, density-dependent reductions in aphid lifespan were strongest on A. viridis (Fig. 4b, host $\times$ density: $\left.F_{6,196}=8.22, P<0.0001\right)$. As density increased aphid mass at maturity decreased on $A$. viridis, but not on the other two plant species (Fig. $4 \mathrm{c}$, host $\times$ density: $F_{6,196}=2.68, P$ $=0.034$ ). Mass at maturity on A. tuberosa could not be measured because the scale was not accessible at the time of that experiment. Age at maturity was constant over all three densities on A. tuberosa, but it decreased as density increased on the other three species (Fig. 4 d, host $\times$ density: $F_{6,196}=2.14, P=$ $0.05)$. There were no alates produced at any density on $A$. incarnata and $A$. syriaca. However, the proportion alate increased with density on A. tuberosa and A. viridis (Fig. 4e, host $\times$ density: $\left.F_{6.196}=26.49, P<0.0001\right)$. There were alates produced at the highest density on $A$. tuberosa and at the intermediate and highest densities on A. viridis. Total fecundity of clone Florida was $5.65 \%$ lower than that on the other clones (data not shown: $F_{2,196}=3.83, P=0.023$ ). Aphid clone did not influence any other vital rates.

\section{Experiment 4}

When both maternal and offspring densities were varied consecutively, within-generation effects on $A$. nerii vital rates were stronger. In all cases except for proportion alate, the expression of maternal effects did not depend on the offspring environment. Total fecundity in the offspring generation was not influenced (a)

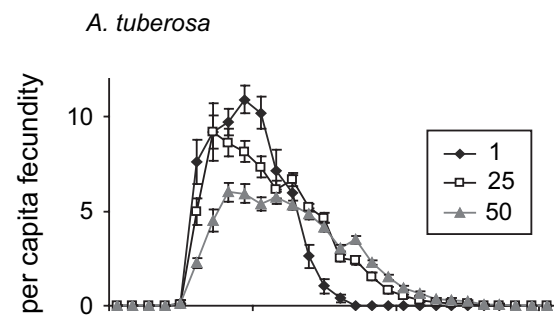

(b)

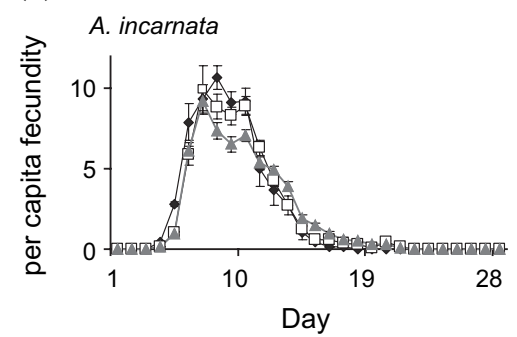

(c)

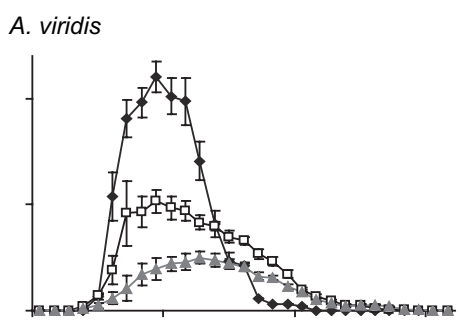

(d)

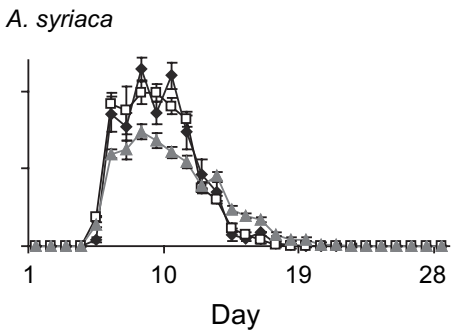

Fig. 3. Effects of density $(1,25,50$ aphid per plant) on Aphis nerii fecundity on four species of Asclepias (a-d). Results from the three clones were combined. Each point is the mean of 21 replicates \pm standard error. 
Fig. 4. Effects of Asclepias species and aphid density on Aphis nerii fecundity (a), lifespan (b), mass at maturity (c), age at maturity (d) and proportion alate (e). Values are means of 21 replicates \pm standard error.

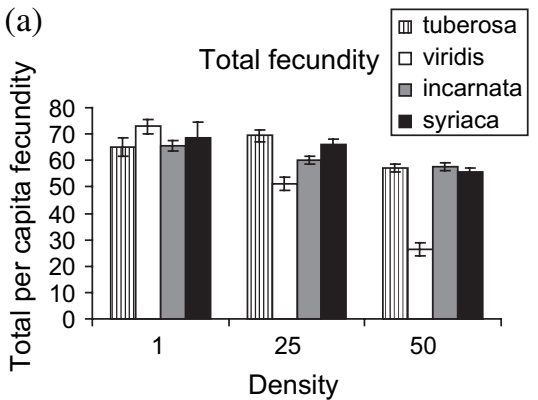

(d)
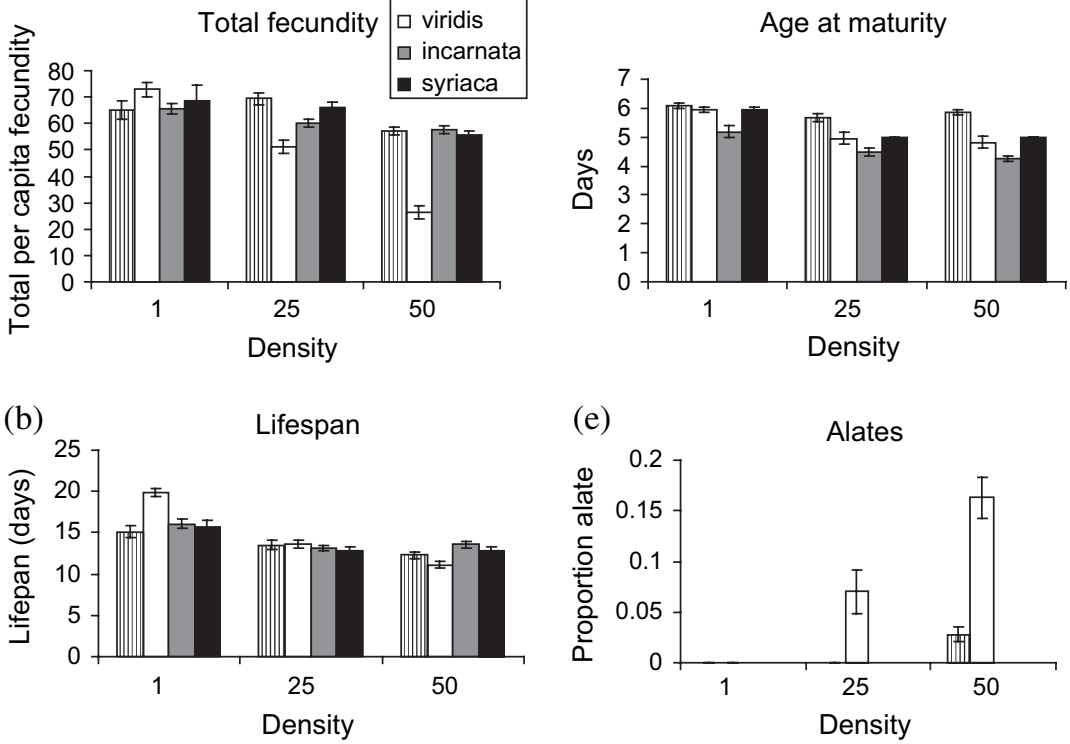

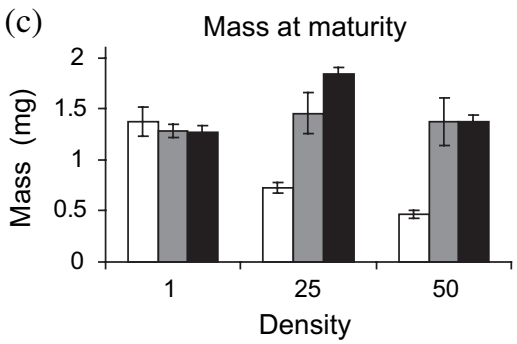

significantly by maternal density, offspring density, or their interaction (Fig. 5a: maternal: $F_{1,33}=3.80, P=0.06$; offspring: $F_{1,33}=3.75, P=0.061$; maternal $\times$ offspring: $F_{1,33}=2.19$, $P=0.148)$. High offspring density, but not maternal density or their interaction, reduced offspring survival (Fig. 5b: maternal: $F_{1,33}=0.17, P=0.682$; offspring: $F_{1,33}=25.79, P<0.0001$; maternal $\times$ offspring: $F_{1,33}=0.12, P=0.731$ ). Similar to experiments 2 and 3 , age at maturity increased with density in the maternal generation, but decreased with density in the offspring generation (Fig. 5c: maternal: $F_{1,33}=5.74, P=0.022$; offspring: $F_{1,33}=32.36, P<0.0001$; maternal $\times$ offspring: $\left.F_{1,33}=0.12, P=0.733\right)$. Alates were produced on only six of 40 plants and five of these were $\mathrm{H}_{\text {maternal }} \mathrm{H}_{\text {offspring }}$ plants (Fig. $5 \mathrm{~d}$ : maternal: $F_{1,33}=7.20, P=0.011$; offspring: $F_{1,33}=9.03, P=$ 0.005 ; maternal $\times$ offspring: $F_{1,33}=7.20, P=0.011$ ). Neither maternal density, offspring density, nor their interaction explained any of the variation in aphid mass at maturity (data not shown; maternal: $F_{1,33}=1.98, P=0.169$; offspring $F_{1,33}=0.15, P=$ 0.701; maternal $\times$ offspring: $\left.F_{1,33}=0.001, P=0.958\right)$.

\section{Discussion}

This research examined the effects of plant quality, insect clone, and insect density on aphid vital rates. The importance of these factors was systematically assessed in both the maternal and offspring generation. While maternal effects were present, within-generation effects on vital rates were much stronger and are therefore more likely to have an impact on aphid population dynamics.

Compared to within-generation effects, maternal effects were relatively weak and had little or no impact on aphid vital rates. Neither maternal density nor maternal host-plant species influenced offspring survival, fecundity, or alate formation. However, maternal density influenced the age at which offspring reached maturity. Offspring of mothers experiencing a low density reached maturity faster, approximately $9 \mathrm{~h}$ earlier, than offspring of high-density mothers. Whether a 9-h difference in development rate matters ecologically for an organism that lives on average for less than 20 days is unclear. Using daily survivorship and fecundity to calculate $r$ for each maternal density treatment showed that these small changes in development schedules influence $r$ and therefore have the potential to influence longterm population dynamics. However, aphids only occur at these low densities (one aphid per plant) when they are colonising a new plant, and then their densities rapidly increase. Currently, it is unknown how these rapid changes in density would influence $r$. Long-term experiments would be required to asses the importance of this effect on the dynamics of A. nerii.

In many published cases, maternal effects are strong initially but then decline as offspring age (Mousseau \& Fox, 1998). 
(a)

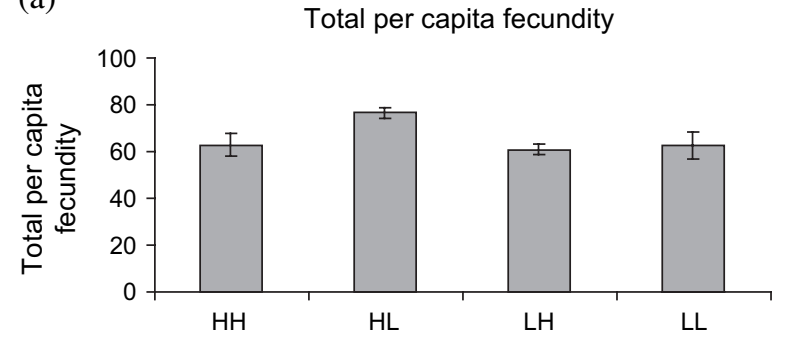

(b)

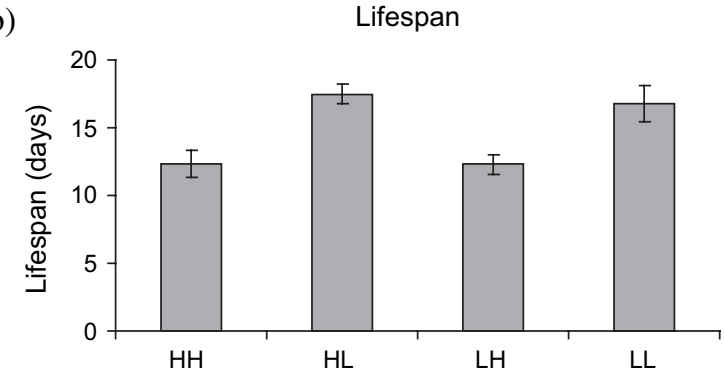

(c)

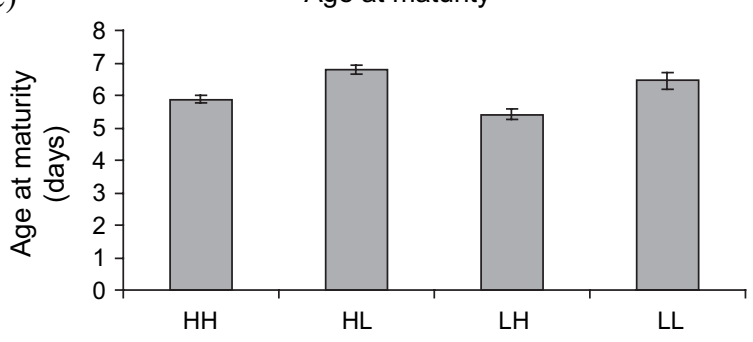

(d)

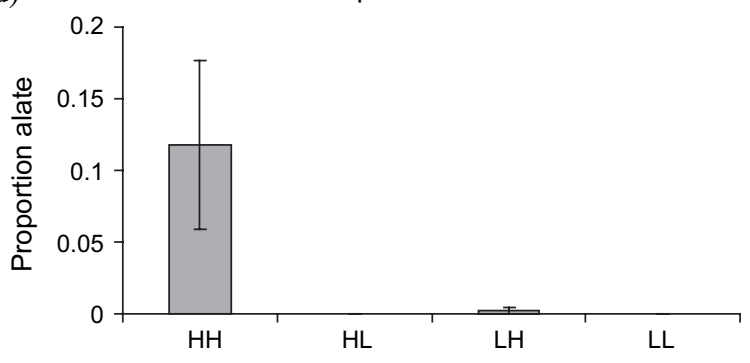

Fig. 5. Effects of maternal and offspring density on offspring fecundity (a), lifespan (b), age at maturity (c), and proportion alate (d) in Aphis nerii. For the $x$-axis labels, the first letter refers to the maternal density and the second letter refers to the offspring density $(\mathrm{H}$, high $=50$ aphids per plant; $\mathrm{L}$, low $=1$ aphid per plant). Values are means of 10 replicates \pm standard error.

Therefore, it was not surprising that maternal density influenced offspring age at maturity, but not offspring survival or fecundity. In a review of environmental influences of diapause expression in insects, Mousseau and Dingle (1991) reported that most maternal effects link environmental cues experienced by mothers with phenotypic expression in embryos, and that plastic responses are generally strongest between adjacent developmental stages (Mousseau \& Dingle, 1991). Therefore, adult traits may not be influenced as strongly by the maternal environment as are embryonic or early developmental traits.

Maternal effects on offspring development rate are not uncommon. Maternal and paternal photoperiod influence offspring development rate in Drosophila melanogaster (Giesel, 1988). Parents experiencing short-day conditions produce progeny with shorter development times than do long-day parents. The host-plant species that a gypsy moth mother is feeding on influences offspring development rate. Offspring develop significantly faster when their mothers feed on black oak than they do on red oak (Rossiter, 1991a).

Originally, it was hypothesised that maternal effects would be a strong force in aphid population dynamics. This hypothesis was based on the fact that aphids have telescoping generations; therefore there is a wide degree of physiological overlap of generations. Additionally, maternal effects are already known to play an important role in determining whether aphids become alate or non-winged, or reproduce sexually or parthenogenetically (Dixon, 1998). Given this evidence, it was thought likely that maternal effects would also influence aphid offspring vital rates. Why then is there an almost complete absence of maternal influence on offspring birth and death rates? Aphids can respond quickly to their environment. For example, some species use environmental cues perceived as first and second instars to determine wing development (Muller et al., 2001) causing cues from the maternal environment to be unnecessary. Maternal effects may be stronger in organisms that are unable to respond as quickly to their environment and must rely on cues from previous generations.

Additionally, an aphid's environment is highly variable. Density of conspecifics can increase from zero to hundreds within 2 weeks (Helms et al., 2004) leading to concomitant changes in plant quality and food availability. Conversely, a strong rainstorm can cause rapid population decline. The environment that an aphid mother experiences may not be an accurate predictor of her offspring's environment. In contrast, maternal effects may be stronger when the maternal environment is a reliable indicator of the offspring's environment.

While across-generation effects appear weak in A. nerii population dynamics, within-generation effects are strong. Aphids exhibited density-dependent survival, fecundity, and alate formation (Figs 3, 4, and 5). On all host-plant species, aphids at low density had higher survivorship during the first half of the experiment (Fig. 3). Low density aphids also had higher per capita fecundity (Fig. 4). This is likely a result of reduced competition for food resources at low density. Densitydependent survival and fecundity has been documented in other experiments (Harrison \& Cappuccino, 1995), as well as in time series analysis. Agrawal (2004), detected negative densitydependence of aphids on 17 of the 18 milkweed species he studied, although the strength of density-dependence varied across species.

In this study, production of alates increased with increasing aphid density (Fig. 5e). This is a common response to high density in aphids and it has been documented in many species (Muller et al., 2001). 
The comparisons of aphid vital rates among plant species in experiment 3 should be treated with caution because these experiments were in different months. Nonetheless, results varied among the different host-plant species. On three of the four plant species (A. incarnata, A. viridis, and A. syriaca), total fecundity decreased as density increased (Fig. 4a). However, on A. tuberosa there was no significant difference in total fecundity between the high and low densities, and the intermediate density actually exhibited the highest total fecundity. The densitydependent reduction in fecundity was much stronger on $A$. viridis than on any of the other Asclepias species, with the largest difference in total fecundity between the low and high density treatments (Fig. 4a).

Similarly, Agrawal (2004) demonstrated density-dependent fecundity of $A$. nerii on different species of milkweed and the strength of this effect depended on host-plant species. A number of plant quality traits, including cardenolide concentration and trichome density, were found to be correlated with the intrinsic rate of aphid population increase. Other studies have documented changes in herbivore population dynamics that are related to differences in plant quality. For example, bean beetle population dynamics vary depending on soybean genotype (Underwood \& Rausher, 2000). For spirea aphids, development time, survival, and fecundity depend on host-plant species (Tsai \& Wang, 2001).

In this study, variation in vital rates among aphid clones was only directly examined in one set of experiments (experiment 3). Clone Florida produced, on average, $5.65 \%$ fewer aphids than clone Augusta or Emory. Whether this translates into differences in population growth rates under field conditions is currently unknown. On only one host-plant species, A. tuberosa, was there a significant clone effect on per capita fecundity. While these clones were collected from geographically distinct locations, it is possible that they are identical genotypes, or that there is no clonal variation in survival and fecundity among the clones that we studied. Similarly, no clonal variation in survival and fecundity was found among 12 populations of the corn leaf aphid collected in Canada (Simon et al., 1995).

The offspring environment can interact with the maternal environment and influence the strength of maternal effects (Rossiter, 1991b). In this study, the only instance where there was an interaction between the maternal and offspring environment was in the case of alate formation (Fig. 5d). The proportion alate was much higher when both the mothers and offspring experienced high densities. When there was high density in one generation and low density the next, such as in experiment 2 , then alates were not produced. This indicates that the cue for alate production may be compounded over generations. A similar phenomenon is seen in locusts (Islam et al., 1994).

In experiment 4, there was no effect of maternal density, offspring density, or their interaction on total per capita fecundity (Fig. 5a). Based on the earlier maternal density experiment (experiment 2), the lack of maternal density influence was expected. However, based on experiment 3 , it was expected that offspring density would affect total fecundity. It is unknown why the results of the two experiments differed. In experiment 4 (as in experiment 3) aphids experiencing higher densities lived shorter lives irrespective of the maternal density. Likewise, age at maturity was influenced by across- and within-generation effects, similar to results of prior experiments. Offspring age at maturity decreased when their mothers were at low density or if offspring were at high density. Perhaps low density mothers have increased access to resources and produce offspring that reach maturity more quickly and possibly experience a competitive advantage over later-maturing offspring. Maternal allocation decisions can directly influence offspring age/mass at maturity and also indirectly affect the competitive environment that the offspring experience (Benton et al., 2005). Additionally, offspring environment is predicted to influence offspring mass and age at maturity (Plaistow et al., 2004). Plaistow et al. (2004) found that offspring age at maturity decreased in a high food environment. Perhaps the high density of aphids created a phloem sink, which led to an increase in per capita food availability.

These results, like the few others that have compared both within- and across-generation effects on vital rates (Miao et al., 1991; Crone, 1997), show that within-generation effects are stronger. Even though maternal effects have the potential to affect offspring vital rates and long-term population dynamics, this potential is not always met. Aphids can respond quickly to their highly variable environment. Therefore, in this system, cues in the maternal environment, at least those studied here, are not as important for vital rates as cues in the current environment. It is concluded that conditions in the current environment should have greater impacts on population dynamics.

\section{Acknowledgements}

We thank B. Ball, W. Duncan, C. Frost, M. C. Hall, and anonymous reviewers for their helpful comments on the manuscript. We thank P. Doty and S. Scott for laboratory assistance. This work was funded by NSF grant DEB-0342750 to M.D.H.

\section{References}

Agrawal, A.A. (1999) Induced responses to herbivory in wild radish: effects on several herbivores and plant fitness. Ecology, 80, $1713-1723$.

Agrawal, A.A. (2004) Plant defense and density dependence in the population growth of herbivores. American Naturalist, 164, 113-120.

Agrawal, A.A., Laforsch, C. \& Tollrian, R. (1999) Transgenerational induction of defenses in animals and plants. Nature, 401, 60-63.

Beckerman, A., Benton, T.G., Ranta, E., Kaitala, V. \& Lundberg, P. (2002) Population dynamic consequences of delayed life-history effects. Trends in Ecology and Evolution, 17, 263-269.

Benton, T.G., Plaistow, S.J., Beckerman, A.P., Lapsley, C.T. \& Littlejohns, S. (2005) Changes in maternal investment in eggs can affect population dynamics. Proceedings of the Royal Society of London. Series B: Biological Sciences, 272, 1351-1356.

Benton, T.G., Ranta, E., Kaitala, V. \& Beckerman, A.P. (2001) Maternal effects and the stability of population dynamics in noisy environments. Journal of Animal Ecology, 70, 590-599.

Berryman, A.A. (1999) Principles of Population Dynamics and Their Application. Stanley Thornes (Publishers) Ltd, Cheltenham, UK.

Crone, E.E. (1997) Parental environmental effects and cyclical dynamics in plant populations. American Naturalist, 150, 708-729.

Del Campo, M.L., Via, S. \& Caillaud, M.C. (2003) Recognition of hostspecific chemical stimulants in two sympatric host races of the pea aphid Acyrthosiphon pisum. Ecological Entomology, 28, 405-412. 
Diss, A.L., Kunkel, J.G., Montgomery, M.E. \& Leonard, D.E. (1996) Effects of maternal nutrition and egg provisioning on parameters of larval hatch, survival and dispersal in the gypsy moth, Lymantria dispar 1. Oecologia, 106, 470-477.

Dixon, A.F.G. (1998) Aphid Ecology: an Optimization Approach, 2nd edn. Chapman \& Hall, London.

Dixon, A.F.G. \& Agarwala, B.K. (1999) Ladybird-induced life-history changes in aphids. Proceedings of the Royal Society of London. Series B: Biological Sciences, 266, 1549-1553.

Fischer, K., Brakefield, P.M. \& Zwaan, B.J. (2003) Plasticityin butterfly egg size: why larger offspring at lower temperatures? Ecology, 84, 3138-3147.

Fox, C.W., Waddell, K.J. \& Mousseau, T.A. (1995) Parental host-plant affects offspring life-histories in a seed beetle. Ecology, 76, 402-411.

Giesel, J.T. (1988) Effects of parental photoperiod on development time and density sensitivity of progeny in Drosophila melanogaster. Evolution, 42, 1348-1350.

Ginzburg, L.R. \& Taneyhill, D.E. (1994) Population-cycles of forest Lepidoptera - a maternal effect hypothesis. Journal of Animal Ecology, 63, 79-92.

Gotelli, N.J. (2001) A Primer of Ecology. Sinauer Associates, Inc., Sunderland, Massachusetts.

Groeters, F.R. \& Dingle, H. (1989) The cost of being able to fly in the milkweed oleander aphid, Aphis nerii (Homoptera, Aphididae). Evolutionary Ecology, 3, 313-326.

Haggstrom, H. \& Larsson, S. (1995) Slow larval growth on a suboptimal willow results in high predation mortality in the leaf beetle Galerucella lineola. Oecologia, 104, 308-315.

Hales, D.F., Wilson, A.C.C., Sloane, M.A., Christophesimon, J., Legallic, J.F. \& Sunnucks, P. (2002) Lack of detectable genetic recombination on the $\mathrm{X}$ chromosome during the parthenogenetic production of female and male aphids. Genetical Research, 79, 203-209.

Hall, R.W. \& Ehler, L.E. (1980) Population ecology of Aphis nerii Homoptera, Aphididae on oleander. Environmental Entomology, 9, 338-344.

Hardie, J. \& Lees, A.D. (1983) Photoperiodic regulation of the development of winged gynoparae in the aphid, Aphis fabae. Physiological Entomology, 8, 385-391.

Harrison, S. \& Cappuccino, N. (1995) Using density manipulation experiments to study population regulation. Population Dynamics (ed. by N. Cappuccino and P. W. Price). Academic Press, San Diego, California.

Helms, S.E., Connelly, S.J. \& Hunter, M.D. (2004) Effects of variation among plant species on the interaction between a herbivore and its parasitoid. Ecological Entomology, 29, 44-51.

Hockham, L.R., Graves, J.A. \& Ritchie, M.G. (2001) Variable maternal control of facultative egg diapause in the bushcricket Ephippiger ephippiger. Ecological Entomology, 26, 143-147.

Hunter, M.D. (2002) Maternal effects and the population dynamics of insects on plants. Agricultural and Forest Entomology, 4, 1-9.

Islam, M.S., Roessingh, P., Simpson, S.J. \& McCaffery, A.R. (1994) Effects of population-density experienced by parents during mating and oviposition on the phase of hatchling desert locusts, Schistocerca gregaria. Proceedings of the Royal Society of London. Series B: Biological Sciences, 257, 93-98.

Kendall, B.E., Ellner, S.P., McCauley, E., Wood, S.N., Briggs, C.J., Murdoch, W.M. et al. (2005) Population cycles in the pine looper moth: dynamical tests of mechanistic hypotheses. Ecological Monographs, 75, 259-276.

Ladner, D.T. \& Altizer, S. (2005) Oviposition preference and larval performance of North American monarch butterflies on four Asclepias species. Entomologia experimentalis et applicata, 116, 9-20.

Lees, A.D. (1963) The role of photoperiod and temperature in the determination of parthenogenetic and sexual forms in the aphid Megoura viciae Buckton. 3. Further properties of the maternal switching mechanism in apterous aphids. Journal of Insect Physiology, 9, 153-164.

Lill, J.T. \& Marquis, R.J. (2001) The effects of leaf quality on herbivore performance and attack from natural enemies. Oecologia, 126, 418-428.

Littell, R.C., Henry, P.R. \& Ammerman, C.B. (1998) Statistical analysis of repeated measures data using SAS procedures. Journal of Animal Science, 76, 1216-1231.

Malcolm, S.B., Cockrell, B.J. \& Brower, L.P. (1989) Cardenolide fingerprint of monarch butterflies reared on common milkweed, Asclepias syriaca. Journal of Chemical Ecology, 15, 819-853.

Miao, S.L., Bazzaz, F.A. \& Primack, R.B. (1991) Effects of maternal nutrient pulse on reproduction of 2 colonizing Plantago species. Ecology, 72, 586-596.

Mousseau, T.A. \& Dingle, H. (1991) Maternal Effects in Insects: Examples, Constraints, and Geographic Variation. Dioscorides Press, Portland, Oregon.

Mousseau, T.A. \& Fox, C.W. (1998) Maternal Effects as Adaptations. Oxford University Press, New York, New York.

Muller, C.B., Williams, I.S. \& Hardie, J. (2001) The role of nutrition, crowding and interspecific interactions in the development of winged aphids. Ecological Entomology, 26, 330-340.

Nunes, M.V. \& Hardie, J. (1999) The effect of temperature on the photoperiodic 'counters' for female morph and sex determination in two clones of the black bean aphid, Aphis fabae. Physiological Entomology, 24, 339-345.

Plaistow, S.J., Lapsley, C.T., Beckerman, A.P. \& Benton, T.G. (2004) Age and size at maturity: sex, environmental variability and developmental thresholds. Proceedings of the Royal Society of London. Series B: Biological Sciences, 271, 919-924.

Rossiter, M. (1988) Relationships among defoliation, red oak phenolics, and gypsy moth growth and reproduction. Ecology, 69, 267-277.

Rossiter, M.C. (1991a) Environmentally-based maternal effects - a hidden force in insect population dynamics. Oecologia, 87, 288-294.

Rossiter, M.C. (1991b) Maternal effects generate variation in lifehistory - consequences of egg weight plasticity in the gypsy moth. Functional Ecology, 5, 386-393.

Rossiter, M. (1994) Maternal effects hypothesis of herbivore outbreak. Bioscience, 44, 752-763.

Simon, J.C., Hebert, P.D.N., Carillo, C. \& Demelo, R. (1995) Lack of clonal variation among Canadian populations of the corn leaf aphid, Rhopalosiphum maidis Fitch (Homoptera, Aphididae). Canadian Entomologist, 127, 623-629.

Tsai, J.H. \& Wang, J.J. (2001) Effects of host plants on biology and life table parameters of Aphis spiraecola (Homoptera: Aphididae). Environmental Entomology, 30, 44-50.

Underwood, N. \& Rausher, M.D. (2000) The effects of host-plant genotype on herbivore population dynamics. Ecology, 81, 1565-1576.

Via, S. (1991) The genetic structure of host plant adaptation in a spatial patchwork - demographic variability among reciprocally transplanted pea aphid clones. Evolution, 45, 827-852.

Wainhouse, D., Ashburner, R. \& Boswell, R. (2001) Reproductive development and maternal effects in the pine weevil Hylobius abietis. Ecological Entomology, 26, 655-661.

Wheeler, G.S. (2001) Host plant quality factors that influence the growth and development of Oxyops vitiosa, a biological control agent of Melaleuca quinquenervia. Biological Control, 22, 256-264.

Wiktelius, S. (1992) The induction of alatae in Rhopalosiphum padi (L.) (Hom., Aphididae) in relation to crowding and plant-growth stage in spring sown barley. Journal of Applied Entomology, 114, 491-496.

Accepted 27 July 2006 